ARTICLE

https://doi.org/10.1038/s41467-020-20757-1

\title{
Nanobody-mediated control of gene expression and epigenetic memory
}

\author{
Mike V. Van (1) ${ }^{1}$, Taihei Fujimori (i) ${ }^{2} \&$ Lacramioara Bintu (i] ${ }^{2 凶}$
}

Targeting chromatin regulators to specific genomic locations for gene control is emerging as a powerful method in basic research and synthetic biology. However, many chromatin regulators are large, making them difficult to deliver and combine in mammalian cells. Here, we develop a strategy for gene control using small nanobodies that bind and recruit endogenous chromatin regulators to a gene. We show that an antiGFP nanobody can be used to simultaneously visualize GFP-tagged chromatin regulators and control gene expression, and that nanobodies against HP1 and DNMT1 can silence a reporter gene. Moreover, combining nanobodies together or with other regulators, such as DNMT3A or KRAB, can enhance silencing speed and epigenetic memory. Finally, we use the slow silencing speed and high memory of antiDNMT1 to build a signal duration timer and recorder. These results set the basis for using nanobodies against chromatin regulators for controlling gene expression and epigenetic memory.

\footnotetext{
${ }^{1}$ Department of Biology, Stanford University, Stanford, CA 94305, USA. ${ }^{2}$ Department of Bioengineering, Stanford University, Stanford, CA 94305, USA.

凶email: Ibintu@stanford.edu
} 
C ontrolling gene expression levels and epigenetic memory is critical for dissecting biological processes, such as development and cancer, and for developing synthetic biology applications that rely on engineering human cells. As a result, the development of tools that can target a specific gene and alter its expression in a controlled manner is crucial for the advancement of this field. Typically, these tools consist of one or more effector domains from transcription factors or chromatin regulators (CRs), fused to a DNA-binding domain that can bind to a specific sequence (e.g., Tetracycline Repressor or Gal4, mostly used as components of synthetic genetic circuits) or be engineered to target any sequence in the genome (e.g., Zinc Fingers, TALEs, or dCas9).

In mammalian cells, the most efficient way to control gene expression and impart epigenetic memory is to engage multiple CRs in a combinatorial fashion. For example, the Krüppelassociated box (KRAB), a strong repressive domain commonly used in gene circuits and CRISPR interference (CRISPRi) applications ${ }^{1}$, recruits KAP1, which in turn interacts with other repressors such as histone deacetylases (HDACs) via the NuRD complex $^{2}$, histone methylase SETDB1, and heterochromatic protein $\mathrm{HP}^{3}$. Previous works have also shown that combining other CRs, such as MeCP2 and DNMT3A, with KRAB can further improve its silencing strength and epigenetic memory. Adding MeCP2 increases silencing strength at certain endogenous genes ${ }^{4}$, whereas adding DNMT3A can increase silencing duration after a short initial pulse of gene targeting ${ }^{5-7}$. Further transcriptional control via histone deacetylation ${ }^{8}$, acetylation ${ }^{9}$, methylation ${ }^{10}$, and demethylation ${ }^{11}$ by CRs fused with dCas 9 has also been reported, highlighting the flexibility of these tools in imparting diverse epigenetic modifications.

Most CRs are large, consisting of multiple domains that are necessary for their function, either to stimulate catalytic function or to mediate interactions with other members of the complexes they are part of. For example, HP1's role in the formation of transcriptionally inactive heterochromatin is dependent on its two protein domains, its chromodomain (CD) and an evolutionary related chromoshadow domain (CSD). The CD allows $\mathrm{HP} 1$ to bind to $\mathrm{H} 3 \mathrm{~K} 9$ methylated chromatin and is required for its gene silencing function ${ }^{12,13}$, whereas the CSD helps HP1 bind to other HP1-like proteins ${ }^{14}$ and other interactors such as SUV39H1 and DNA methyltransferases ${ }^{15}$, which may further enhance these silencing effects. Furthermore, sometimes the catalytic domain of a $\mathrm{CR}$ is not sufficient for silencing. Unlike DNMT3A and DNMT3B, the C-terminal catalytic domain of DNMT1 has been shown to be catalytically incapable of methylating DNA by itself ${ }^{16,17}$, requiring a large part of the $\mathrm{N}$-terminal domain for its activity ${ }^{18}$. Therefore, to obtain efficient gene control, it is often desirable to recruit full-length CRs to a target gene.

Due to the large size of CRs and the requirement to use them in combinations, it is difficult to build compact gene regulatory tools that can fit in viral delivery vectors such as adeno-associated virus (AAV) (payload limit $\leq 4.7 \mathrm{~kb}$ ) or lentivirus (payload limit $\leq 9.7 \mathrm{~kb}$ ). This problem is further exacerbated by the size of Streptococcus pyogenes Cas9, which at over $4.2 \mathrm{~kb}$ makes adding one or more CRs challenging. To overcome this size limit, a smaller variant of dCas 9 has been engineered by deleting various functional domains; and when combined with a small transactivation domain was able to barely fit within the packaging limit of AAV and showed efficient activation activity ${ }^{19}$. In addition, splitting the dCas9 protein (e.g., by utilizing two dimerizable fragments ${ }^{20}$ or the intein-mediated trans-splicing system ${ }^{21,22}$ ) or simply separating the dCas9 protein and guide RNAs into two different viral delivery vectors have been used to overcome this packaging limit. However, these latter approaches can suffer from poor editing efficiency due to the inability to deliver all payload components into one given cell. Therefore, although there are current efforts to find and test smaller CRISPR variants (reviewed in ref. ${ }^{23}$ ), it is equally important to develop smaller effector domains.

Here we take an approach to building smaller tools for gene regulation: instead of fusing large CRs to the DNA-binding domain, we use single-domain antibodies (also called nanobodies) to recruit endogenous CRs from the existing cellular chromatin network. Due to their small size $(\sim 15 \mathrm{kDa})$, strong binding affinity, and high stability, nanobodies have been utilized successfully in many research applications, including live-cell imaging, protein immunoprecipitation, and fluorescent protein enhancement (reviewed in refs. ${ }^{24,25}$ ). We show that nanobodies can be used to silence gene expression and impart epigenetic memory, and can enhance the function of commonly used transcriptional effectors. Using nanobodies to recruit endogenous CRs to a gene of interest for transcriptional control could offer a better opportunity to recruit entire chromatin complexes, taking advantage of all domains essential for activity and their interactions.

\section{Results}

Nanobodies against GFP-CRs are used to control gene expression. To test whether nanobodies can be used to recruit CRs for efficient gene expression control, we started with a well-characterized nanobody against green fluorescent protein $(\mathrm{GFP})^{26-28}$. We fused this nanobody to a reverse tetracycline repressor (rTetR) DNA-binding domain and used it to recruit various publicly available GFP-tagged CRs (GFP-CR) to a TagRFP fluorescent reporter gene located at the AAVS1 locus in HEK293T cells (Fig. 1a). This reporter contains five Tet operator (TetO)-binding sites upstream of a constitutive $\mathrm{pEF}$ promoter driving the expression of the fluorescent gene. Upon the addition of doxycycline (dox) to the cell media, rTetR binds to these sites, allowing us to control recruitment and release of the rTetRnanobody fusion at the reporter.

Using this system, we first used time-lapse microscopy to measure the localization dynamics of GFP-HP1a and GFPHDAC5 during transient expression, and their connection to gene expression in a cell population stably expressing the rTetRantiGFP nanobody fusion and the TagRFP reporter. We observed that both GFP-tagged HP1 $\alpha$ and HDAC5 mediated fast silencing of the reporter, as indicated by the decrease in TagRFP signal as early as $24 \mathrm{~h}$ after dox addition (Fig. 1b). Furthermore, we found that although HP1a localizes mostly in the nucleus (Supplementary Fig. 1a), HDAC5 was seen translocating in and out of the nucleus (Supplementary Fig. 1b), as previously reported ${ }^{29}$. In cells where there was dilution of the GFP expression vector and thus the expression of the CR was lost, the TagRFP reporter reactivated after $\sim 50 \mathrm{~h}$ (Fig. 1b). At the end of 5 days of recruitment (dox treatment), the TagRFP reporter was still silent in cells that were still expressing GFP (Fig. 1c).

Based on these promising results, we then used flow cytometry to measure reporter silencing after 5 days of recruitment in cells ectopically expressing GFP-HP1 $\alpha$, GFP-HP1 $\beta$, GFP-HP1 $\gamma$, or GFPHDAC5. By the end of 5 days of transient expression and recruitment, all CRs tested were able to silence the reporter in a dox-dependent manner in the majority of the cells still expressing the GFP-CR (Fig. 1d and Supplementary Fig. 1c). In addition, recruitment of GFP alone does not lead to silencing of the reporter, which suggests the silencing observed is dependent on the activity of the recruited CR (Fig. 1d). HP1 $\alpha$, HP1 $\beta$, and HDAC5 lead to a higher fraction of cells silenced compared to $\mathrm{HP} 1 \gamma$, consistent with their reported roles in silencing and association with 
a

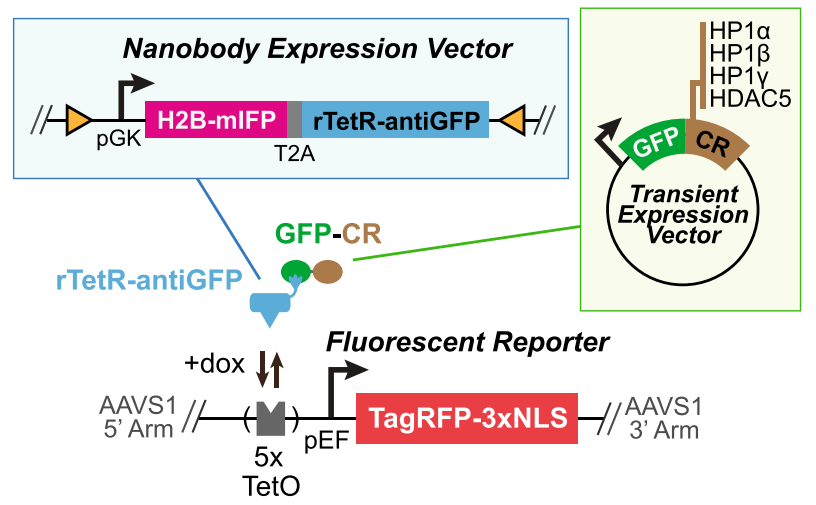

d

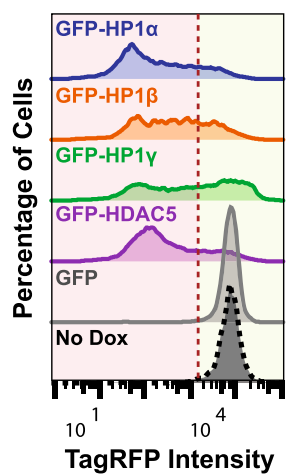

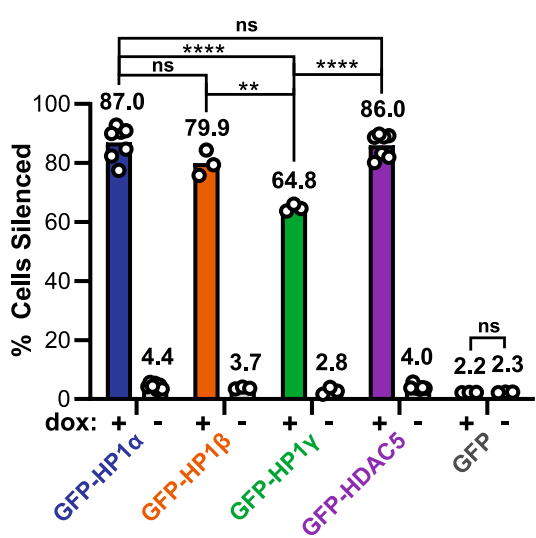

C

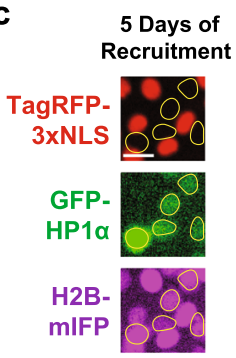

b

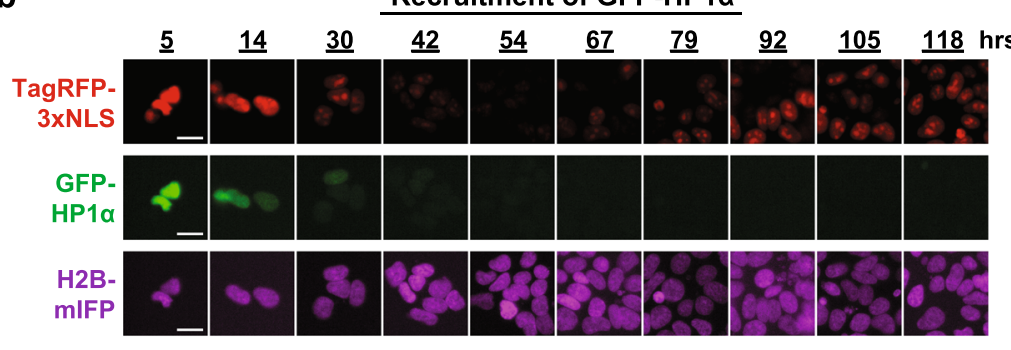

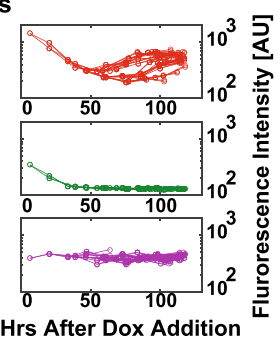

Hrs After Dox Addition

Recruitment of GFP-HDAC5
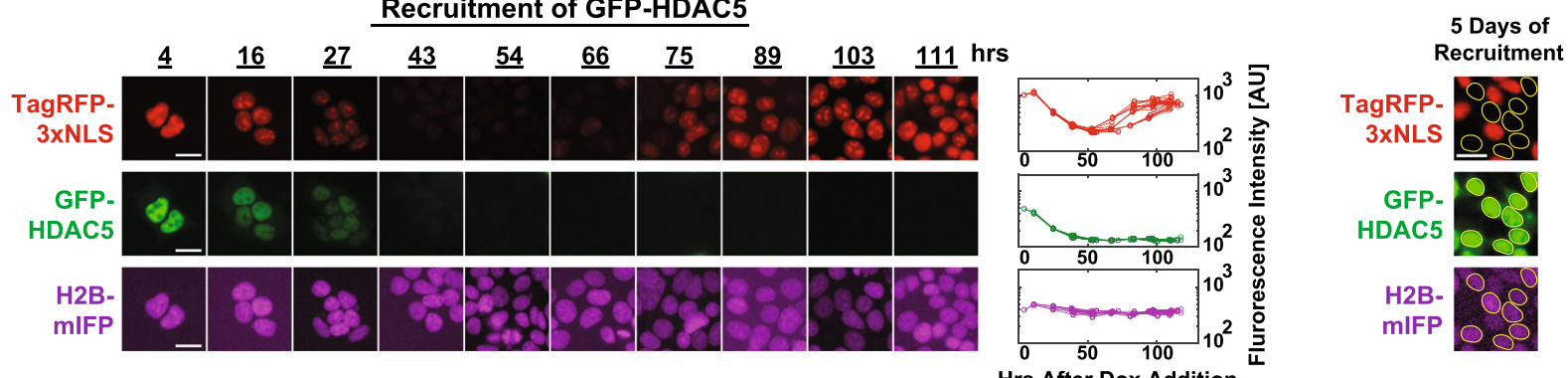

Fig. 1 Nanobodies against GFP-tagged chromatin regulators allow for gene expression control. a Construct for constitutive coexpression (under the pGK promoter) of H2B-mIFP and antiGFP nanobody fused with the rTetR DNA-binding protein (separated by the self-cleaving peptide T2A) was randomly integrated into HEK293T cells by PiggyBac (blue box, top). These cells also contain a TagRFP reporter gene integrated at the AAVS1 "safe harbor" locus and driven by the pEF promoter (bottom). Five TetO-binding sites allow binding of rTetR upstream of the reporter gene upon dox addition. The nuclear localization signal (NLS) and $\mathrm{H} 2 \mathrm{~B}$ domains localize fluorescent protein signals to the nucleus, improving quantification during time-lapse imaging. Plasmids expressing GFP-tagged CRs (HP1 $\alpha, \mathrm{HP} 1 \beta, \mathrm{HP} 1 \gamma$, and HDAC5) were transiently transfected into cells (green box, right). b Time-lapse imaging of cells upon recruitment of GFP-tagged HP1 $\alpha$ (top) and HDAC5 (bottom). Cells stably expressing the reporter (TagRFP, red) and the rTetR-antiGFP fusion (mIFP, purple) were transiently transfected with GFP-HP1 $\alpha$ or GFP-HDAC5 (GFP, green). Cells were treated with dox throughout the movie starting at time 0 hours. Example images across the time-lapse movie (left) and single-cell traces for individual cell lineages as they undergo up to eight cell divisions (right). c Cells that still have GFP-CR expression (yellow circles) by day 5 of recruitment remain silenced. Images and analysis in $\mathbf{b}$ and $\mathbf{c}$ from one biological replicate. Scale bars: $20 \mu \mathrm{m}$. d Left: fluorescence distributions measured by flow cytometry showing reporter silencing after recruitment of GFPCRs ( + dox) for 5 days. Cells were gated for the presence of both GFP-CR (GFP positive) and rTetR-antiGFP (mIFP positive). The red dotted line was used to determine the percentage of cells silenced shown on the right. Right: mean percentage of cells silenced upon presence or absence of dox for 5 days. Each dot is an independently transfected biological replicate (GFP-HP1 $\alpha: n=7$; GFP-HP1 $\beta: n=3$; GFP-HP1 $\gamma: n=3$; GFP-HDAC5: $n=7$; GFP: $n=3$ ). Statistical analysis by two-tailed Tukey's test (GFP-HP1 $\alpha$ vs. GFP-HP1 $\gamma:{ }^{\star \star \star \star} p=4.8 \mathrm{e}-6$; GFP-HP1 $\beta$ vs. GFP-HP1 $\gamma:{ }^{\star \star} p=0.0031 ;$ GFP-HP1 $\gamma$ vs. GFP-HDAC5: ${ }^{\star \star \star \star} p=$ $9.0 e-6)$. Source data are available in the Source Data file.

heterochromatin ${ }^{30,31}$. Nevertheless, HP1 $\gamma$, which can associate with either heterochromatin ${ }^{32}$ or actively transcribed regions ${ }^{33,34}$, still leads to silencing in a majority of cells (Fig. 1d). Surprisingly, lower levels of GFP-CRs led to a higher fraction of cells silenced after 4 days of recruitment for all four CRs (Supplementary Fig. 1d). This observation suggests that CR levels are not the limiting factor for silencing and lower levels may be in fact better. Moreover, increasing the number of antiGFP nanobodies fused to a single rTetR to 8 did not increase the fraction of cells silenced
(Supplementary Fig. 1e), suggesting that a single nanobody is sufficient for silencing in our reporter system.

Nanobodies against DNMT1 and HP1 can silence a reporter gene and confer epigenetic memory. Encouraged by the results with the antiGFP nanobody, we proceeded to test two existing nanobodies against endogenous CRs, antiHP1 $1^{35}$ and antiDNMT1 ${ }^{36,37}$, for their capacity to silence and induce epigenetic memory. The antiHP1 
nanobody was shown to bind to all three isoforms of HP1 in cell lysate by western blotting and in cells by immunofluorescence ${ }^{35}$. The antiDNMT1 nanobody has been used to immunoprecipitate endogenous DNMT1 from whole cell lysate ${ }^{36}$ and visualize endogenous DNMT1 in live cells ${ }^{37}$. We cloned fusions between rTetR and either antiHP1 or antiDNMT1, and stably integrated them into HEK293T cells containing the TagRFP reporter with TetO sites at the AAVS1 locus (Fig. 2a). Both nanobodies mediated doxdependent silencing upon recruitment at the reporter for 5 days, albeit weaker than KRAB (Fig. 2b), a known strong repressor ${ }^{38}$ used in CRISPRi ${ }^{1}$. Although KRAB can strongly silence nearly all (97.9\%) of the cells, antiHP1 and antiDNMT1-mediated silencing were weaker, resulting in $16.8 \%$ and $28.5 \%$ of cells silenced, respectively (Fig. 2b). Surprisingly, silencing by antiHP1 is weaker than GFPtagged HP1 proteins when mediated by the antiGFP nanobody ( $16.8 \%$ vs. $64 \%$ to $87 \%$ depending on the HP1 isoform recruited, Fig. 1d). This difference in silencing is not due to low levels of endogenous HP1 in the cell, as overexpression of HP1a did not enhance silencing by the antiHP1 nanobody (Supplementary Fig. 1f). This finding is in agreement with the observation above that CR levels are not limiting (Supplementary Fig. 1d) and point to other possible causes that limit the antiHP1 silencing efficiency, such as nanobody-binding kinetics.

As DNMT1 is considered a maintenance DNA methylase that usually methylates hemi-methylated DNA, and has lower catalytic activity on unmethylated DNA compared to de novo methyltransferases DNMT3A and DNMT3B ${ }^{16,17}$, we wondered whether antiDNMT1-mediated silencing is associated with DNA methylation. To study the effects of antiDNMT1 recruitment on DNA methylation, we analyzed the CpG methylation state of the reporter via targeted bisulfite sequencing ("Methods"). Due to antiDNMT1's weak silencing of the reporter after 5 days of dox, we decided to sort silenced cells (TagRFP-negative; +dox OFF) from non-silenced (TagRFP-positive, + dox ON) cells (Supplementary Fig. 2a) to measure any differences in methylation between these two cell populations. Recruitment of antiDNMT1 resulted in higher levels of methylated CpGs at the reporter in the sorted OFF population when compared to the ON population in the dox-treated cells, to the no dox cells (Fig. 2c), and to the negative controls at the actin locus and unmethylated DNA (Supplementary Fig. 2b). The DNA methylation levels with antiDNMT1 were comparable to direct DNMT1 recruitment (Fig. 2c), but lower than those obtained after recruitment of DNMT3B. Overall, these results show that silencing of the reporter by antiDNMT1 is associated with methylation of $\mathrm{CpG}$ DNA. In addition, treatment of the cells with 5-aza-2'deoxycytidine (5-aza-2'), a DNA methyltransferase inhibitor, during recruitment abolished antiDNMT1 mediated silencing but not KRAB or antiHP1 (Supplementary Fig. 2c), indicating that DNA methylation is required for antiDNMT1-mediated silencing. On the other hand, treatment with chaetocin, a broadspectrum inhibitor of lysine histone methyltransferases, reduced all three effectors' ability to silence (Supplementary Fig. 2d), suggesting that histone methylation is involved in both antiHP1and antiDNMT1-mediated silencing.

After recruiting the effectors for 5 days with dox, we released the cells from dox and assessed epigenetic memory by measuring the percentage of cells still silenced over 30 days with flow cytometry (Fig. 2d). We found that silencing mediated by antiHP1 was almost completely reversible (Fig. 2e; orange line), which is consistent with previous reports that silencing by HP1 is associated with limited and partial memory, with the majority of the cells reactivating gene expression over time ${ }^{30,39}$. In contrast, KRAB and antiDNMT1 led to more permanent memory, with about $50 \%$ and $65 \%$ cells irreversibly silenced out of the total initially silenced, respectively (Fig. 2e; blue and purple lines). These observations were consistent with the reactivation dynamics of KRAB in a different mammalian cell line (CHO cells $)^{40}$ and the role of DNA methyltransferases in mediating epigenetic memory ${ }^{5-7,41}$. Overall, these results demonstrate the first use of a nanobody that can mediate gene silencing and impart epigenetic memory.

In addition to testing the nanobodies individually, we fused the two nanobodies together with a short flexible linker to see if this would enhance gene silencing and memory at the reporter gene (Supplementary Fig. 2e). Recruitment of rTetR-antiDNMT1antiHP1 led to about $80 \%$ of the cells being silenced, which is more than each nanobody individually (Fig. 2b). Moreover, the antiDNMT1-antiHP1 fusion had improved epigenetic memory over KRAB, with $61.4 \%$ cells still silent at 30 days after dox removal vs. $48.6 \%$ cells, respectively (Fig. 2 e; green vs. blue line). These results show for the first time that combining two nanobodies that bind different CRs can be used to enhance gene silencing as well as epigenetic memory, and demonstrates the promising prospect of using nanobodies in combination with other CRs to improve transcriptional control.

Having the option to transiently express these nanobodies and bypass the need to stably integrate them in cells may provide greater flexibility for transcriptional control. Therefore, we tested how effective these nanobodies were at silencing when transiently expressed in our TagRFP reporter cell line. AntiHP1 and antiDNMT1 led to weak silencing of the reporter after 5 days of dox treatment (Supplementary Fig. 3a). Recruitment of antiDNMT1-antiHP1 led to about 55\% of the cells being silenced, which is more than each nanobody individually (Supplementary Fig. 3a), similar to the stable expression results (Fig. 2b). To measure epigenetic memory independent of silencing efficiency, we decided to sort the silenced (TagRFP-negative) cells at the end of 5 days of dox treatment and measure their persistence of silencing for 30 days. Similar to stable expression, we found that the antiDNMT1-antiHP1 fusion had improved epigenetic memory over KRAB, with $35.4 \%$ cells still silent at 30 days post sorting vs. $15 \%$ cells, respectively (Supplementary Fig. 3b). Overall, these results show that transient expression of nanobodies can also lead to reporter silencing and impart epigenetic memory, but less efficiently than stable expression.

Recruitment of antiDNMT1 improves silencing speed and epigenetic memory of other CRs at the reporter. Realizing the potential of using nanobodies in combination with other CRs, we decided to test the antiDNMT1 nanobody in combination with KRAB (Supplementary Fig. 4a). As KRAB has strong, fast silencing, and antiDNMT1 has long-lived epigenetic memory, we hypothesized that combining KRAB and antiDNMT1 might allow us to build a small tool for fast silencing and long-lived memory that can be delivered transiently. Transient expression and recruitment of rTetR-KRAB-antiDNMT1 to the reporter for 5 days resulted in strong silencing $(87.8 \%)$, on the same level as KRAB alone (88.9\%) (Fig. 3a), but demonstrated improved memory (Fig. 3b; pink vs. blue line). This increase is especially important for experiments that involve transient KRAB expression, as epigenetic memory of KRAB when transiently expressed (Fig. 3b; blue line, 15\%) is lower than in cell lines stably expressing KRAB (Fig. 2e; blue line, $48.6 \%$ ), presumably due to the dilution of the plasmid. The further addition of the antiHP1 nanobody to the KRAB-antiDNMT1 fusion did not increase silencing or memory further (Fig. 3a, b; brown).

Moreover, when targeting rTetR-KRAB and rTetR-antiDNMT1 simultaneously at the reporter as stably expressed separate fusions, epigenetic memory was even lower than with KRAB alone (Supplementary Fig. 4b). We measured a similar decrease in memory and silencing upon co-recruitment of separate fusions of 
a

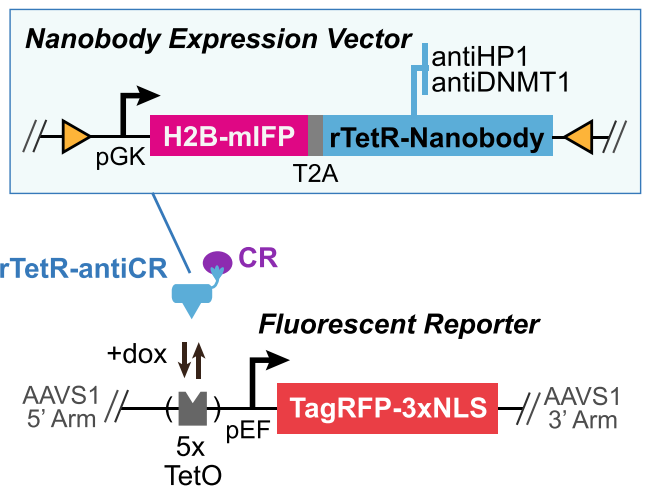

b

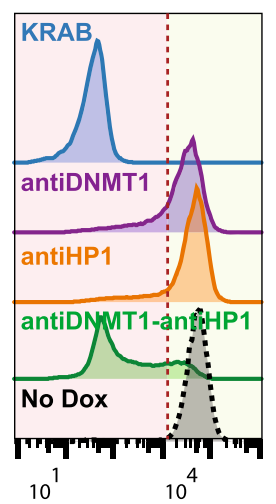

TagRFP Intensity

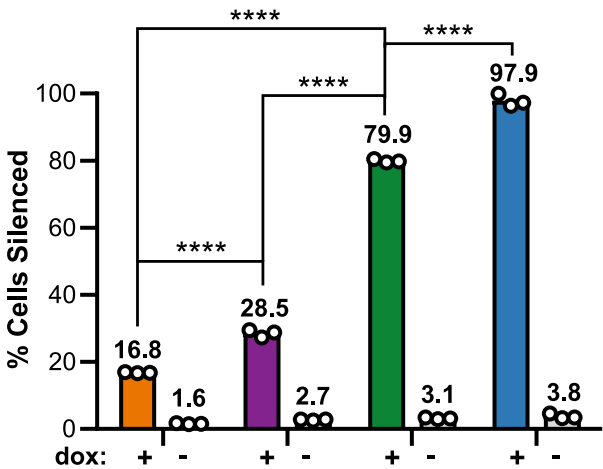

antiHP1 antiDNMT1 antiDNMT1 KRAB - antiHP1

C

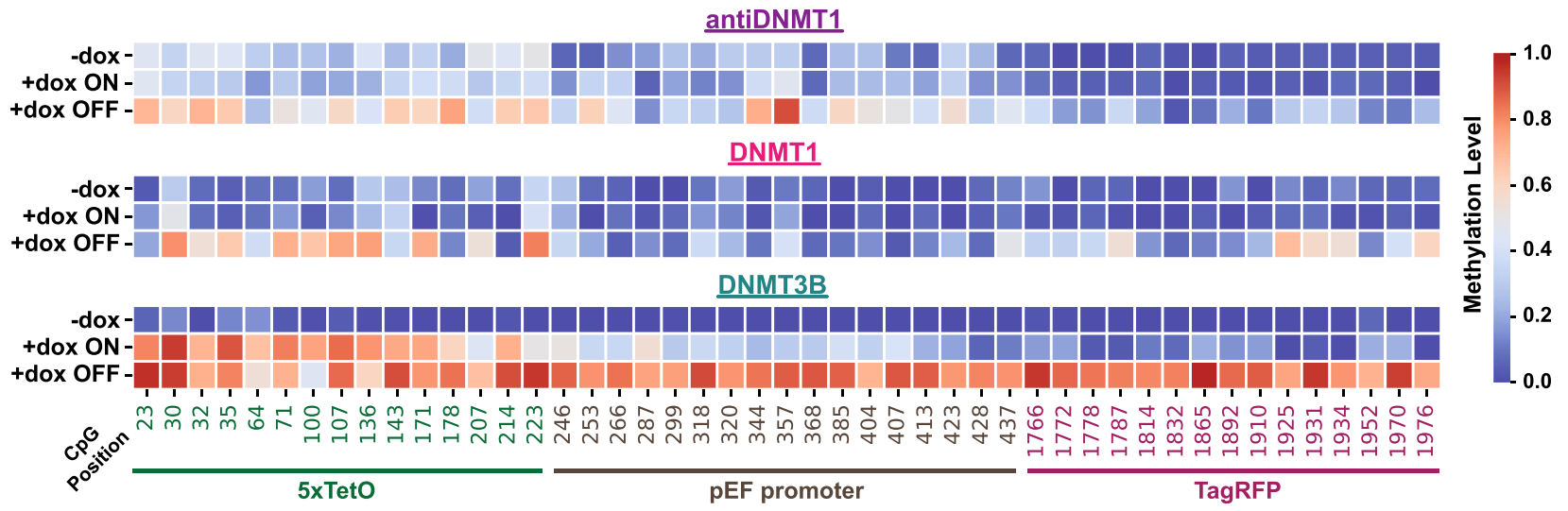

d

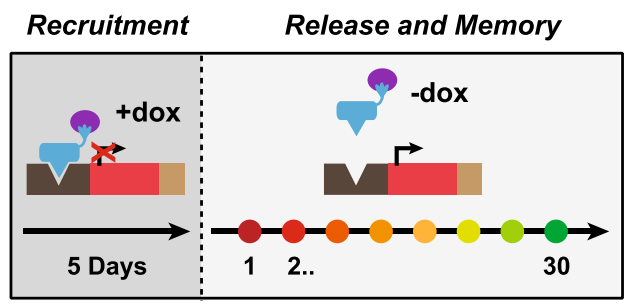

e

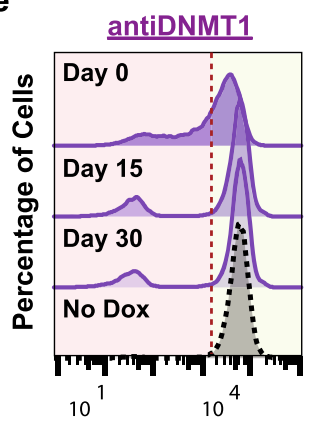

TagRFP Intensity

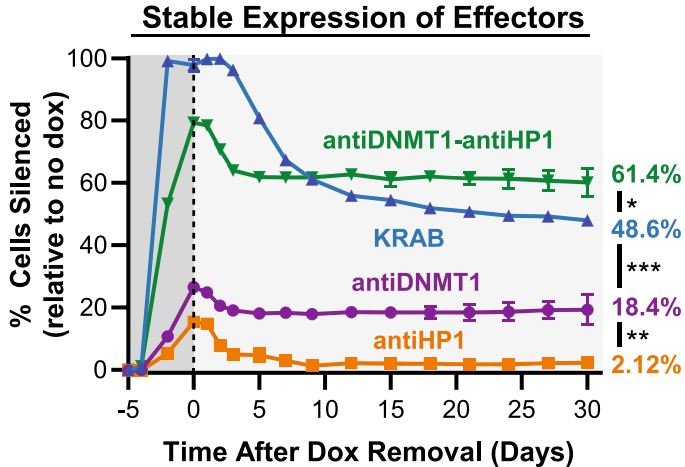

Fig. 2 Nanobody-mediated recruitment of endogenous chromatin regulators can silence gene expression and confer memory. a Construct for constitutive coexpression of H2B-mIFP and nanobody against CR (antiHP1 or antiDNMT1) fused with the rTetR DNA-binding protein (blue box, top) were expressed in HEK293T cells containing a TagRFP reporter (bottom). b Left: fluorescence distributions of the TagRFP reporter after 5 days of recruitment (dox treatment) in cells stably expressing the nanobody constructs or rTetR-KRAB were analyzed by flow cytometry to determine the percentage of cells with the reporter silenced (left of the red dotted line). Right: means of percentages cells silent were calculated from 3 replicates. Statistical analysis by twotailed Tukey's test (antiHP1 vs. antiDNMT1: ${ }^{\star \star \star \star} p=5.6 \mathrm{e}-6$; antiHP1 vs. antiDNMT1-antiHP1: ${ }^{\star \star \star \star} p=2.5 \mathrm{e}-13$; antiDNMT1 vs. antiDNMT1-antiHP1: ${ }^{\star \star \star \star} p=$ 1.8e - 11; antiDNMT1-antiHP1 vs. KRAB: ${ }^{\star \star \star *} p=1.7 \mathrm{e}-7$ ). c Targeted bisulfite sequencing of the reporter after 5 days of recruitment with antiDNMT1, DNMT1, and DNMT3B (+dox), compared to untreated cells expressing the same effectors ( - dox). Dox-treated cells were sorted based on the silencing of the TagRFP reporter labeled as +dox ON and +dox OFF (see Supplementary Fig. 2a for representative gating). CpG positions are calculated relative to the start of the most upstream TetO site. Positive and negative controls for DNA methylation are shown in Supplementary Fig. 2b. d Experimental design for investigating epigenetic memory: rTetR-effectors were recruited to the reporter for 5 days (+dox) and then released ( - dox). Memory was monitored after dox removal via flow cytometry throughout 30 days. e Silencing and memory dynamics data (right) for the experiment described in $\mathbf{d}$ with representative flow cytometry histogram for antiDNMT1 at day 0,15, 30 after dox removal (left). The percentage of cells silenced was normalized to the no dox control to adjust for any background silencing ("Methods"). Means are from three replicates. SDs are plotted but are too small to show for most data points. Statistical analysis by two-tailed Tukey's test at day 30 after dox removal (antiHP1 vs. antiDNMT1: ${ }^{\star \star} p=0.0076$; antiDNMT1 vs. KRAB: ${ }^{\star \star \star} p=0.00026 ;$ KRAB vs. antiDNMT1-antiHP1: ${ }^{\star} p=0.043$ ). Source data are available in the Source Data file. 
TagRFP Intensity

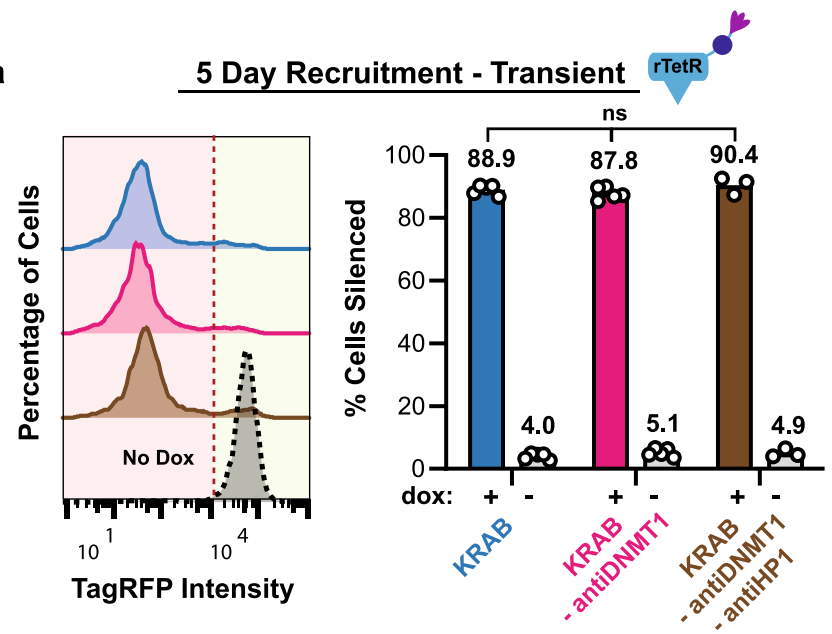

b

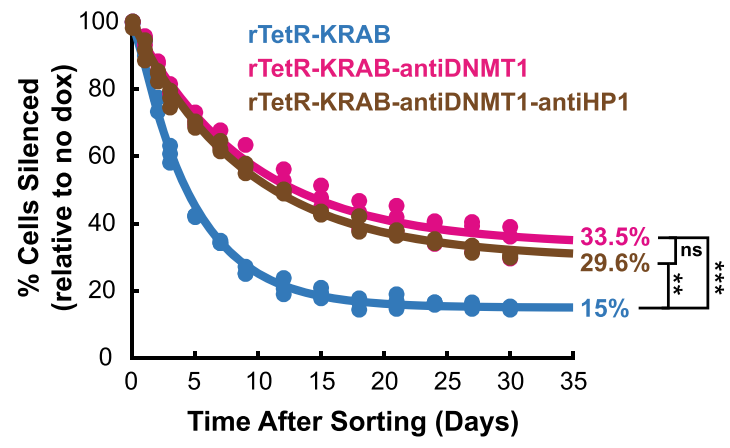

d

C
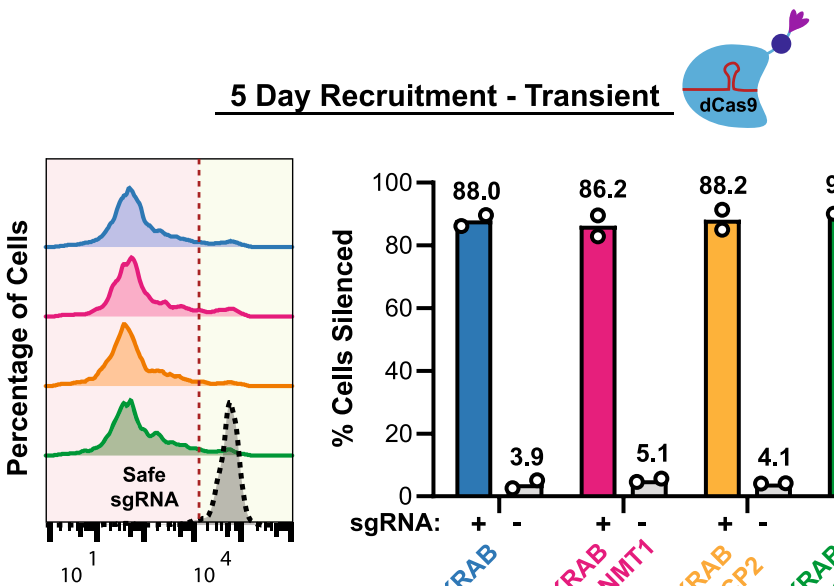

TagRFP Intensity
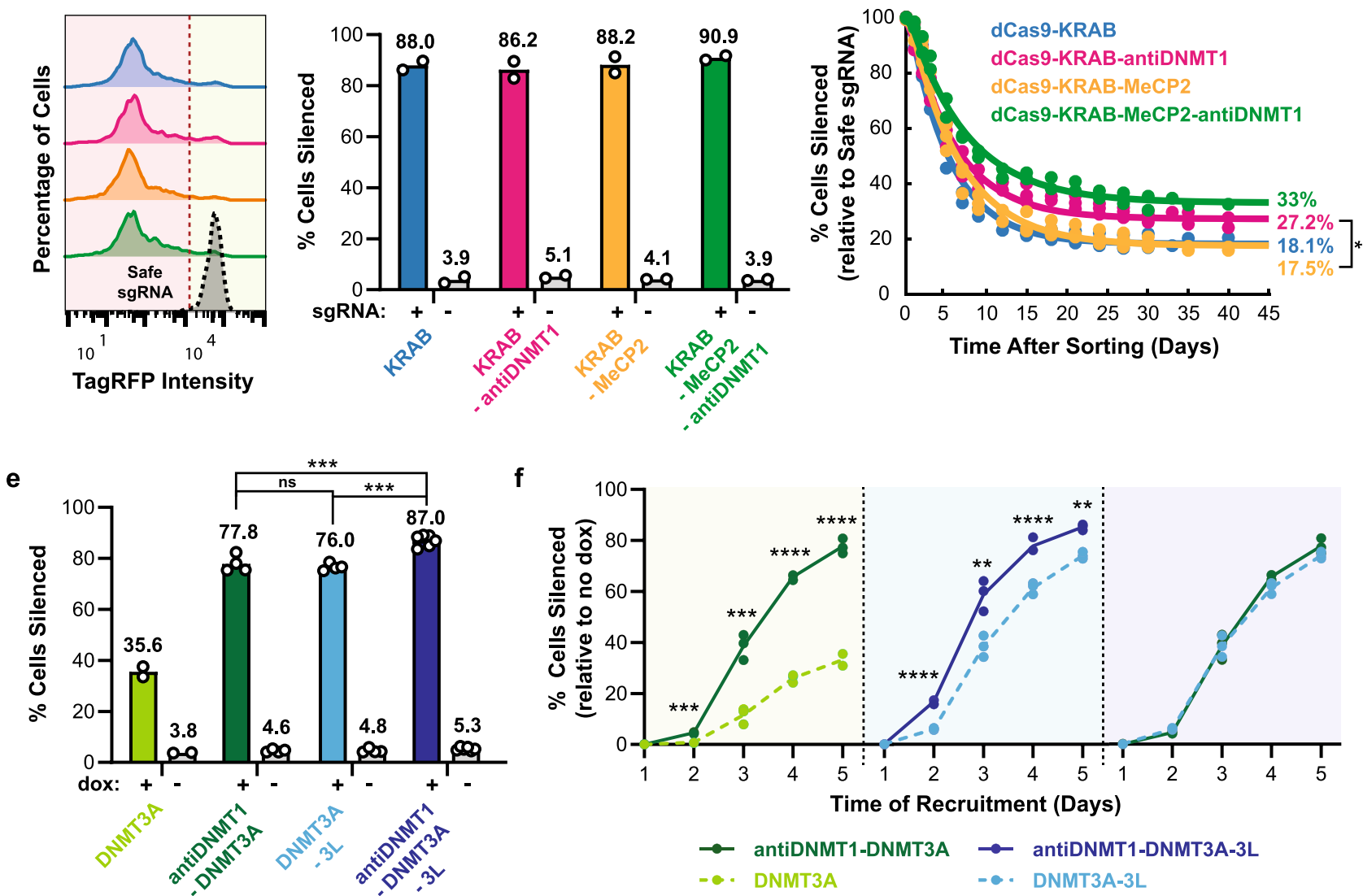

rTetR with antiHP1 and antiDNMT1 (Supplementary Fig. 4b vs. Fig. 2e). These observed reductions in epigenetic memory and silencing may be due to effector binding competition at the five TetO sites and suggest that direct fusions of multiple CRs might be preferable to independent co-recruitment at a locus for gene transcriptional control.

To expand the use of this tool for endogenous gene silencing, we tested the KRAB-antiDNMT1 fusion with the dCas9 system at the reporter gene (Supplementary Fig. 4c). Single-guide RNAs (sgRNAs) were designed to target the $5 \times$ TetO-binding site upstream of the reporter and a genomic site with no annotated function to serve as a negative control (called safe-targeting guide). When the programmable dCas9-KRAB-antiDNMT1 was targeted to the reporter gene for 5 days, there was strong silencing (Fig. 3c) and improvement in memory over dCas9-KRAB (Fig. 3d; $27.2 \%$ vs. $18.1 \%$ ). Recruitment of dCas9-KRAB-antiDNMT1 not only demonstrated improved memory over dCas9-KRAB but also over a newly developed combined repressor, dCas9-KRAB$\mathrm{MeCP}^{4}$, (Fig. 3d; $27.2 \%$ vs. $17.5 \%$ ). In our system, KRAB$\mathrm{MeCP} 2$ has the same memory as KRAB alone (Fig. $3 \mathrm{~d} ; 18.1 \%$ vs. $17.5 \%)$ and the addition of antiDNMT1 to this fusion resulted in a similar improvement in memory as when added to KRAB (Fig. 3d; $33 \%$ for KRAB-MeCP2-antiDNMT1 vs. $27.2 \%$ for KRAB-antiDNMT1). These results suggest that the antiDNMT1 
Fig. 3 Nanobody-mediated enhancement of KRAB and DNMT3A repression. a Left: fluorescence distributions of the reporter gene after transient expression of $r$ TetR-effector fusions and recruitment by dox treatment for 5 days, measured using flow cytometry. Right: the means of percent cells silent (to the left of the red dotted line in the left-side plots) are shown as bars. Each dot represents an independently transfected biological replicate (KRAB: $n=5$; KRAB-antiDNMT1: $n=5$; KRAB-antiDNMT1-antiHP1: $n=3$; statistical analysis by one-way ANOVA). b After recruitment with $r$ TetR-effector fusions for 5 days, silenced cells were sorted and memory dynamics were measured by flow cytometry throughout 30 days. Data represent three biological replicates and are fitted with an exponential decay curve ("Methods"). Two-tailed Tukey's test analysis at day 30 after sorting (KRAB vs. KRABantiDNMT1: ${ }^{\star \star \star} p=0.00022 ; \mathrm{KRAB}$ vs. KRAB-antiDNMT1-antiHP1: $\left.{ }^{\star \star} p=0.0016\right)$. $\mathbf{c}$ Fluorescence distributions after transient expression and targeting of dCas9-effector fusions to the TetO sites upstream of the reporter gene (+) or to a safe-targeting control site $(-)$ for 5 days. Means are from two biological replicates. d After targeting the dCas9-effector fusions for 5 days, silenced cells were sorted, and memory dynamics was measured by flow cytometry throughout 40 days. Each dot is a biological replicate (KRAB: $n=2 ;$ KRAB-antiDNMT1: $n=3 ;$ KRAB-MeCP2: $n=3 ;$ KRAB-MeCP2-antiDNMT1: $n=2$ ) and are fitted with an exponential decay curve ("Methods"). Two-tailed Tukey's test at day 40 after sorting (KRAB-MeCP2 vs. KRAB-antiDNMT1: ${ }^{\star} p=0.040$ ). e Transient expression and recruitment of rTetR-based DNA methyltransferase combinations to the reporter gene for 5 days (DNMT3A: $n=2$; antiDNMT1-DNMT3A: $n=4$; DNMT3A-3L: $n=4$; antiDNMT1-DNMT3A-3L: $n=6$ ). Statistical analysis by two-tailed Tukey's test (antiDNMT1-DNMT3A vs. antiDNMT1-DNMT3A-3L: ${ }^{\star \star \star} p=0.00044$; DNMT3A-3L vs. antiDNMT1-DNMT3A-3L: $\left.{ }^{\star \star \star} p=0.00015\right)$. f Corresponding silencing dynamics of $r T e t R-$ based combinations in e throughout 5 days of recruitment $(n=3$ replicates). Statistical analysis by two-tailed Tukey's test (DNMT3A vs. antiDNMT1DNMT3A: ${ }^{\star \star \star} p_{2}=0.00023,{ }^{\star \star \star} p_{3}=0.00051,{ }^{\star \star \star \star \star} p_{4}=4.3 \mathrm{e}-8,{ }^{\star \star \star \star \star} p_{5}=3.5 \mathrm{e}-7 \mid$ DNMT3A-3L vs. antiDNMT1-DNMT3A-3L: ${ }^{\star \star \star \star} p_{2}=5.3 \mathrm{e}-8$,

$\left.{ }^{\star \star} p_{3}=0.0033,{ }^{\star \star \star \star} p_{4}=5.1 \mathrm{e}-5,{ }^{\star \star} p_{5}=0.0019\right)$. Source data are available in the Source Data file.

nanobody can be used to enhance the permanent silencing ability of CRs. However, when we targeted the dCas9-KRABantiDNMT1 fusion to the CXCR4 endogenous gene, we did not observe an increase of epigenetic memory compared to dCas9KRAB alone (Supplementary Fig. 4d, e), suggesting that the KRAB-antiDNMT1 tool requires further systematic characterization with respect to genomic locus and promoter type. Although the level of memory seen after rTetR-KRAB-antiDNMT1 recruitment at the reporter is smaller than previously observed with the triple combination KRAB-DNMT3A-Dnmt3L $\mathrm{L}^{5,7}$, KRAB-antiDNMT1 is about three times smaller (Supplementary Fig. $4 \mathrm{a} ; \sim 580 \mathrm{bp}$ vs. $\sim 1770 \mathrm{bp}$ ) and thus may be a more appealing tool for viral-based methods.

Recruitment of the catalytic domain of DNMT3A at a gene locus can induce DNA methylation and stable gene repression ${ }^{42,43}$. However, as DNMT3A alone typically leads to slow transcriptional repression, it is common to combine it with other CRs to enhance its effects ${ }^{5-7}$. Realizing the potential of the antiDNMT1 nanobody in improving the gene repressive effects of $K R A B$, we wanted to test whether combining the nanobody with the catalytic domain of DNMT3A would enhance it as well. When the rTetR-antiDNMT1DNMT3A fusion was recruited to the reporter gene via rTetR for 5 days, it led to stronger and faster silencing when compared to DNMT3A alone (Fig. 3e, f; dark green vs. light green). In addition, it has been shown that DNMT3L can enhance the catalytic activity of DNMT3A $\mathrm{A}^{44-46}$. Consistent with previous work, the addition of the C-terminal domain of mouse Dnmt3L and the catalytic domain of DNMT3A enhanced silencing of our reporter from 35.6 to 76 percent (Fig. 3e; light blue). Surprisingly, the addition of the smaller antiDNMT1 nanobody to DNMT3A led to a similar improvement in silencing as the larger Dnmt3L domain (Fig. 3e, f; dark green vs. light blue). The antiDNMT1 nanobody further improved silencing when added to the DNMT3A-3L fusion (Fig. 3e, f; dark blue vs. light blue). In fact, of the different rTetR fusion combinations tested, the antiDNMT1-DNMT3A-3L triple fusion was by far the strongest (Fig. 3e; dark blue) resulting in about $87 \%$ of the cells being silenced at 5 days of dox. In summary, the antiDNMT1 nanobody improved the speed of silencing in all combinations with DNMT3A (Fig. 3f). All fusions containing rTetR-DNMT3A, including the ones containing antiDNMT1, led to permanent epigenetic memory at our reporter gene (Supplementary Fig. 5a). We also see a similar increase in the speed of silencing of the reporter gene when we fused antiDNMT1 to the HDAC enzyme HDAC4 (Supplementary Fig. 5b). These promising results suggest the fusion of a small antiDNMT1 nanobody to CRs may serve as a way to enhance silencing or memory.
Nanobody-mediated recruitment of CRs for synthetic circuit control. These nanobody-based tools for controlling gene expression and epigenetic memory could serve as devices in synthetic circuits for detecting and recording signals. Cellular stopwatches and recording devices are important components of synthetic biology circuits ${ }^{47}$. The response of the antiDNMT1 nanobody presents a unique opportunity of implementing a very compact stopwatch that can both measure and record the duration of a signal. The desired signal can be coupled to the expression of rTetR-antiDNMT1, which in turn can be recruited upstream of an output gene encoding for fluorescence, signaling molecules, or proteins involved in cell death or survival (Fig. 4a). The addition of dox starts the time recording session, while removal of dox ends it. As silencing by antiDNMT1 recruitment is quite slow, it leads to a linear response in the percentage of cells silent as a function of signal duration (Fig. 4b). Moreover, as antiDNMT1 recruitment leads to permanent memory in the majority of cells silenced, the signal duration can be recorded as a percentage of cells in the population that have the output gene off (Fig. 4c). Coupling antiDNMT1 expression or recruitment to another cellular signal (such as a cytokine or a hormone) would allow one to measure and permanently record the total duration of that signal as the fraction of cells with the reporter silenced.

The linear response to signal duration and its recording in the fraction of irreversibly silent cells can be described using a 3-state model of gene control (Fig. 4d, "Methods") 40 . In this model, active cells (A) silence at a slow rate $\left(k_{\mathrm{S}}\right)$ during recruitment by antiDNMT1. They first reach a reversible silent state $(R)$ and can transition from this to an irreversibly silent state $(I)$ with a rate $k_{\mathrm{I}}$. After release of the nanobody, the reversibly silent population reactivates at a rate $k_{A}$, while the irreversibly silent cells remain silent. Experimentally, we also observed a delay between the addition of dox and the onset of silencing, so we incorporated this lag time in the model as an additional parameter: $T_{\text {lag }}$ (Supplementary Note and "Methods"). By fitting the experimental data for antiDNMT1 silencing and reactivation of the 14day time course in Fig. 4c ("Methods"), we can extract the three rates, the lag time before onset of silencing, and their $95 \%$ confidence intervals: $k_{\mathrm{S}}=0.11 /$ day $(0.1-0.12), k_{\mathrm{A}}=0.29 /$ day $(0.12-0.45), k_{\mathrm{I}}=0.37 /$ day $(0.31-0.43), T_{\mathrm{lag}}=1.7$ days $(1.3-2.0)$. As the exponential rate of silencing is quite low, it results in an approximately linear fraction of cells silenced as the duration of signal (dox recruitment) increases (Fig. 4b, c, red dots). Similarly, the fraction of irreversibly silent cells that record signal duration looks approximately linear with signal duration for a large range of signals (Fig. 4e). 
a

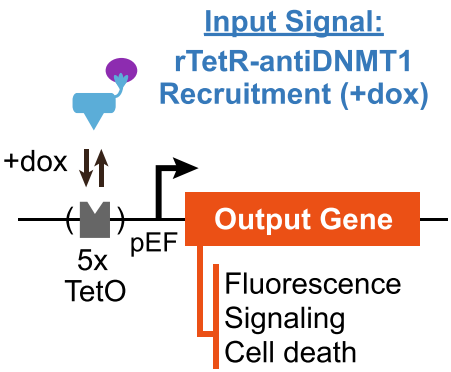

c
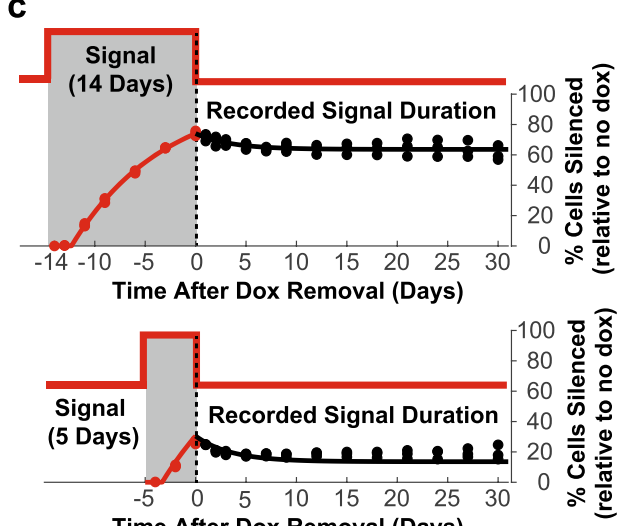

Time After Dox Removal (Days) b

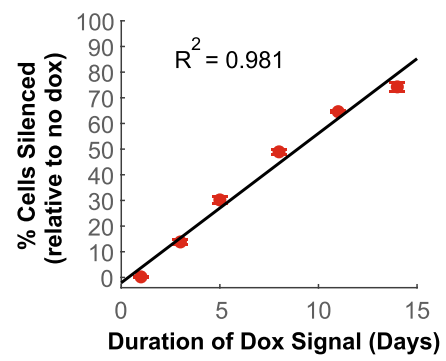

d

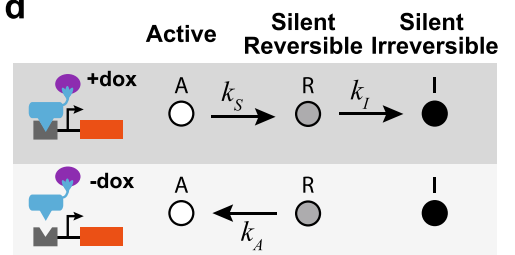

e

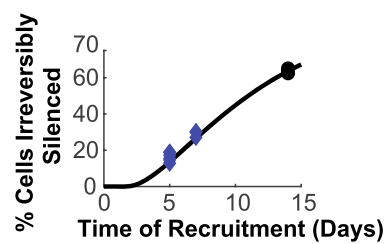

f

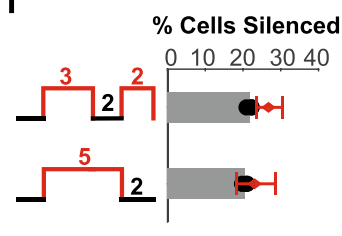

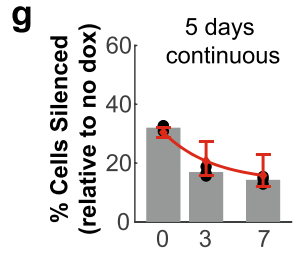

Fig. 4 Nanobodies as signal detection and recording tools. a Schematic of a device for measuring and recording signal duration. The input signal is coupled to the recruitment of rTetR-antiDNMT1 near the pEF promoter to silence an output gene. $\mathbf{b}$ Percentages of cells with TagRFP reporter silenced as measured by flow cytometry at the end of the indicated dox signal durations in a cell line stably expressing rTetR-antiDNMT1. Means and SDs of experimental data from three replicates (red dots) and linear fit (black, "Methods"). c Percentage of cells with TagRFP silenced after different signal (dox treatment) durations: 14 days (top) and 5 days (bottom). The gray shaded regions (negative numbers) indicate the period with dox. Dashed lines indicate dox removal. Continuous lines represent fits to the model in $\mathbf{d}$ for the 14 days data and predictions of the model for the 5-day data ("Methods" and Supplementary Note). d Three-state model of silencing by antiDNMT1 during recruitment (+dox, top) and during release ( - dox, bottom). e Percentage of cells irreversibly silent after different durations of recruitment (dox treatment) predicted by the model in $\mathbf{d}$ are plotted as a black line ("Methods"). Experimental data recorded at 7 days during the release period shown as black dots if used for model fitting (14 days), or blue diamonds if not used in the fit. $\mathbf{f}$ Percentages of cells silenced relative to no dox controls for pulsed recruitment (top: 3 days + dox, 2 days - dox, 2 days + dox) compared to continuous recruitment for the same duration (bottom: 5 days +dox, 2 days -dox). Experimental data from three replicates shown as black dots, means as gray bars, and model predictions with $95 \% \mathrm{Cl}$ in red ("Methods"). $\mathbf{g}$ Percentages of cells silenced relative to no dox controls for pulsed recruitment vs. continuous recruitment, recorded at the same time after dox removal, plotted as in $\mathbf{f}$. Source data are available in the Source Data file.

Once calibrated, the three-state phenomenological model can be used to predict the fraction of cells silent over time for different types of signals without changing the three transition rates and initial lag time. For example, the model predicts that the fraction of cells silenced at the end of a 5-day pulsed signal ( 3 days of dox, 2 days of no dox, and then 2 days of dox) is approximately the same as at the end of a continuous 5-day signal ( 5 days of dox and then 2 days of no dox), matching experimental data (Fig. 4f). This model also predicts that continuous signals result in a small but systematically higher level of cells permanently silenced compared to interrupted signals of the same total duration, which is also consistent with experimental data (Fig. 4g). It is noteworthy that for pulsed signals there are two start times of dox addition, so there are two lag time values. For all our model predictions in Fig. 4f, g, we set the first lag time to the value obtained from fitting the 14-day continuous time course in Fig. $4 \mathrm{c}\left(T_{\mathrm{lag} 1}=1.7\right)$, but we assumed that there is no delay between dox addition and silencing on the second dox pulse $\left(T_{\mathrm{lag} 2}=0\right)$. Although this assumption slightly overestimates the percentage of cells silent in the pulsed experiments, it leads to predictions that are closer to experimental data than setting the two lag times equal (Supplementary Fig. 6). The reduction in the second lag time suggests an intricate interplay between DNA methylation and silencing: it is possible that residual methylation from the first dox pulse allows onset of silencing to happen faster on the second pulse $\left(T_{\mathrm{lag}_{2}}<T_{\mathrm{lag} 1}\right)$. Although we still need to understand the connection between DNA methylation and gene silencing better, ultimately this type of modeling can be used to program the fraction of cells active or silenced for synthetic biology applications. 


\section{Discussion}

In this study, we developed a technology that uses nanobodies to recruit endogenous CRs at a target gene for transcriptional and epigenetic memory control in mammalian cells. We showed that the antiGFP nanobody that binds to GFP-CRs can mediate gene silencing when recruited to a reporter gene. Nanobodies against endogenous CRs (antiHP1 and antiDNMT1) can mediate silencing as well. Moreover, these nanobodies led to stronger silencing or more persistent epigenetic memory when combined with each other or with other CRs (KRAB, DNMT3A, and HDAC4). These results highlight the use of nanobodies as a method to enhance gene silencing by combining them with other effectors, and open avenues for development, characterization, and application of compact nanobody-based tools in gene regulation.

Although here we used the antiGFP nanobody mainly for an initial proof-of-concept experiment, to show that targeted recruitment of nanobodies against GFP-CR fusions can mediate gene silencing, this approach can be further extended to elucidate how CR localization and gene expression are interconnected. Different CRs are known to mediate liquid-liquid phase separation and translocate in and out of the nucleus, and the dynamics of these localization are thought to regulate gene expression. For example, HP1 localizes primarily to the nucleus, can form phase separated regions that partition heterochromatin ${ }^{48,49}$, and mediate silencing and partial epigenetic memory when recruited at a gene $^{30}$. Class IIa HDACs $(4,5,7$, and 9) translocate in and out of the nucleus in a cell-cycle-dependent manner and this shuttling phenotype is believed to spatially control their ability to interact with repressive complexes in the nucleus and thus their ability to repress transcription ${ }^{50-52}$. Other localization patterns have been observed for other HDACs as well, ranging from being mostly in one subcellular compartment (nucleus or cytoplasm) to localizing to either compartment depending on the cell type ${ }^{29}$. Our nanobody-based recruitment tool has the potential to be applied to all HDACs (11 total) to better help understand their localization pattern and role in gene regulation changes in different cell types. However, to connect the fast changes in CR localization (shorter than a cell cycle) to gene expression, one needs to use an mRNA-based reporter (e.g., MS2-MCP or PP7-PCP systems) rather than a protein reporter (e.g., mCitrine or TagRFP).

Gene regulation using the antiGFP nanobody can also be used in conjunction with recently engineered cell line collections containing a GFP knock-in at loci expressing specific CRs and transcription factors ${ }^{53,54}$. These cell lines express CR-GFP fusions at endogenous levels, and thus allow characterization of localization, dynamics of phase separation properties, and gene regulation via the antiGFP nanobody at concentrations that are biologically relevant, without overexpression of the regulators. Moreover, some of these cell collections consist of stem cells, either human induced pluripotent cells $^{53}$ or mouse embryonic stem cells ${ }^{54}$, which can be differentiated to different cell types. Therefore, introducing the reporter-antiGFP system in these lines would allow simultaneous characterization of CR and gene regulation dynamics in various differentiated cell types that have the same genetic background without the need for additional cell engineering. Moreover, development of nanobodies against smaller tags, e.g., against the small C-terminal of the split GFP, GFP11, would make this approach easier, as knock-in of GFP11 into endogenous loci can be done in a higher throughput manner ${ }^{55}$. High-affinity nanobodies designed against other small epitopes such as ALFA-tag from an $a$-helical peptide ${ }^{56}$, BC2 SpotTag from $\beta$-catenin ${ }^{57,58}$, EPEA/C-tag from $\alpha$-synuclein ${ }^{59}$, and the UBC6e-tag ${ }^{60}$ may further be useful in in-vivo tracing and recruitment of intracellular CRs.

We also show that nanobodies can be used in synthetic circuits for detecting and recording signals. Using experimental data for
antiDNMT1-mediated silencing and reactivation at the TagRFP reporter, we were able to derive a three-state model of gene control for this nanobody that can be used to predict the fraction of cells silent over time for new types of signals. This interplay between experiment and mathematical modeling is key to informing the design and fine-tuning of synthetic circuits, which can then be applied to engineer larger, more complex networks. In addition, models that accurately predict the behavior of a system can help us engineer cellular outputs without having to perform large numbers of trial-and-error experiments. The framework we developed for antiDNMT1 can be expanded to study the capabilities of other nanobodies and used to optimize gene circuits that take advantage of the specific nanobodies silencing and memory characteristics.

Development of nanobodies against other CRs and transcription factors besides antiHP1 or antiDNMT1 could lead to even more versatile gene regulation and epigenetic control. For example, recruitment of de novo DNA methylases DNMT3A and DNMT3B enables DNA methylation of promoters, enhancers, and other regulatory regions (e.g., CTCF sites) for transcriptional repression $17,42,43,61$. Nanobodies designed against these de novo methylases could be used for locus specific methylation in the same manner, and possibly avoid offtarget methylation toxicity associated with overexpression of the methylases ${ }^{17,62}$. Another class of CR are those associated with euchromatin and gene activation: histone acetylases (CBP, p300 $)^{9}$, DNA hydroxymethylases that mediate removal of DNA methylation (Tet1-3) $42,63,64$, and ATP-dependent chromatin remodeling complexes $(\mathrm{BAF})^{39}$. Nanobodies against these complexes could be used for activation of heterochromatic loci. However, developing nanobodies against co-activators such as CBP or p300 is challenging due to their intrinsically disordered structure that makes them difficult to express homogeneously in vitro ${ }^{65}$. Moreover, when developing nanobodies for gene control, it is important to target CRs that are abundant in the cells of interest and to select nanobodies with high binding affinity to their target CRs. Development of such functional nanobodies that can properly fold in the cellular environment and bind to different chromatin complexes with high affinity, without disrupting their function requires develop of highthroughput screening methods inside human cells. The reporter system and methods described here could serve as a platform for such nanobody functional screens.

To extend the use of nanobodies beyond control of reporter genes, it is necessary to take a systematic high-throughput approach that tests variability with target locus and cell type. Variability in the effect on gene expression is not specific to nanobody-mediated control, but has been reported for direct fusions of dCas9 and full-length CRs ${ }^{5,6}$. For example, persistent epigenetic memory requires both DNA methylation by DNMT3A-3L and histone methylation by EZH2 (H3K27me3) or $\mathrm{KRAB}(\mathrm{H} 3 \mathrm{~K} 9 \mathrm{me} 3)^{6}$. However, which histone methylase is necessary for memory depends on the specific gene targeted ${ }^{5,6}$. Even at genes where KRAB and DNMT3A-DNMT3L corecruitment mediates persistent memory in one cell type, the combination sometimes fails to impart memory in a different cell type ${ }^{5}$. These results suggest we also need a clearer understanding of the co-factors each CR interacts with, and how their expression and activity varies with genomic locus and cell type. Moreover, the off-target effects of different tools can also vary for different dCas 9 fusions ${ }^{66}$, and thus would have to be systematically measured. Compact tools such as nanobodies, which can be more easily cloned and delivered alone and in different combinations, would facilitate systematic measurements of on-target variability and off-target effects of epigenetic editing tools. 


\section{Methods}

Plasmid construction. The TagRFP reporter (5xTetO-pEF-TagRFP-3xNLS) construct was assembled using a AAV zinc finger donor vector backbone (Addgene \#22212) containing a promoter-less splice-acceptor upstream of a puromycin resistance gene and homology arms against the AAVS1 locus. Three elements of the reporter were amplified from the following sources: five TetO-binding sites upstream of a pEF promoter from PhiC31-Neo-ins-5xTetO-pEF-H2B-Citrine-ins (Addgene \#78099),

TagRFP-T from pEN_ERK.KTR-tagRFP-T, and 3xNLS from pEN_mCherry-NLS (both gifts from Joydeb Sinha \& Mary Teruel, Stanford). These components were cloned into the AAV donor vector backbone using Gibson Assembly.

The plasmids containing the rTetR-effector fusions were cloned into the PBCMV-MCS-EF1a-Puro PiggyBac vector backbone (System Biosciences \#PB510B-1), which was further modified via Gibson Assembly with the following components: PGK promoter from pSLQ2818, mIFP from pSLQ2837-1 (both gifts from Tony Gao \& Stanley Qi, Stanford ${ }^{67}$, and H2B-rTetR-Zeo from pEx1-pEF-H2B-mCherry-

T2A-rTetR-KRAB-Zeo (Addgene \#78352). Each effector was PCR amplified from the following plasmid: antiGFP nanobody ${ }^{68}$ - gift from Rajarshi Ghosh \& Jan Liphardt, Stanford; antiDNMT1 nanobody_-purchased from ChromoTek; antiHP1 nanobody ${ }^{35}$-gift from Sandrine Moutel \& Franck Perez, Institut Curie; KRABAddgene \#84241; MeCP2-Addgene \#110821; and DNMT3A-3L-Addgene

\#71827. A list of all CRs used in this study can be found in Supplementary Table 1.

Plasmids containing the dCas9-effector fusions were derived from the dCas9KRAB vector backbone (Addgene \#110820) and modified by Gibson Assembly with their respective effectors from sources listed above. The dCas9-effector fusions containing KRAB or KRAB-antiDNMT1 were further modified with mCitrine-NLS upstream of the dCas9 to allow for cell sorting and analysis of endogenous gene silencing. The sgRNA cloning vector (gift from Josh Tycko \& Michael Bassik, Stanford, Addgene \#89359) was modified to express mIFP or mCherry. Each sgRNA sequence (see Supplementary Table 2) was cloned into the plasmid using the BlpI and BstXI cloning sites, as previously described ${ }^{69}$.

Our AAVS1 TagRFP reporter donor vector (Addgene \#163083) and rTetRantiGFP recruitment vector (Addgene \#163084) have been deposited to Addgene. Plasmids containing antiDNMT1 and/or antiHP1 are available on request upon signing an MTA with ChromoTek and Institut Curie, respectively.

Cell culture. Cells were cultured at $37^{\circ} \mathrm{C}$ under a humidified atmosphere with $5 \%$ $\mathrm{CO}_{2}$. HEK293T cells (Takara Bio \#632180) were maintained in Dulbecco's modified Eagle medium (DMEM; Gibco \#10569010) supplemented with $25 \mathrm{mM}$ Dglucose (Gibco), $1 \mathrm{mM}$ sodium pyruvate (Gibco), $1 \times$ GlutaMAX $^{\mathrm{TM}}$ (Gibco), and $10 \%$ Tet Approved FBS (Clontech Laboratories). When cells reached $80 \%$ confluence, they were gently washed with $1 \times$ DPBS (Life Technologies) and passaged using $0.25 \%$ Trypsin (Life Technologies). For long-term storage, cells were resuspended in freezing media ( $10 \%$ dimethyl sulfoxide (Sigma) and cell media) in a cryovial and frozen at $-80 ;{ }^{\circ} \mathrm{C}$

Stable cell lines construction. The reporter cell line was created by integrating the TagRFP fluorescent reporter at the first intron of the constitutively expressed gene PPP1R12C at the AAVS1 locus in HEK293T cells. The integration of the reporter was performed by co-transfecting $1000 \mathrm{ng}$ TagRFP reporter $(5 \times$ TetO-pEF-TagRFP$3 \times \mathrm{NLS}$ ) donor plasmid and $500 \mathrm{ng}$ of each TALEN arm (AAVS1-TALEN-L (Addgene \#35431) targeting 5'-TGTCCCCTCCACCCCACA-3' and AAVS1TALEN-R (Addgene \#35432) targeting 5'-TTTCTGTCACCAATCCTG-3'). Cells were selected with $500 \mathrm{ng} / \mathrm{mL}$ puromycin (InvivoGen) starting $48 \mathrm{~h}$ post transfection for $\sim 5$ days or until all of the negative control cells died. Cells positive for TagRFP had two peaks representing the monoallelic and bi-allelic integration of the reporter at the AAVS1 locus. Cells with the lower fluorescence peak (monoallelic) were sorted by fluorescence-activated cell sorting using a Sony SH800 Cell Sorter with a $100 \mu \mathrm{m}$ disposable chip. Each of the individual rTetR-effector plasmids was randomly integrated into this reporter line by co-transfecting $250 \mathrm{ng}$ Super PiggyBac Transposase expression vector (System Biosciences \#PB200PA-1) and $750 \mathrm{ng}$ of rTetR-effector donor vector. These cells were selected with $60 \mu \mathrm{g} / \mathrm{mL}$ zeocin (InvivoGen) starting $48 \mathrm{~h}$ post transfection. All transfections were performed in 24well plates using Lipofectamine 2000 (Invitrogen).

Transient transfections. Approximately 70,000 cells were seeded per well in a 24-well plate and the next day cells were transfected using Lipofectamine 2000 (Invitrogen) according to manufacturer instructions. For experiments in the antiGFP nanobody cell line, $1000 \mathrm{ng}$ of each GFP-CR was delivered. For the transient silencing and reactivation experiments, $1000 \mathrm{ng}$ of rTetR-effector expression vector was delivered to each well. $600 \mathrm{ng}$ of dCas9-effector and $400 \mathrm{ng}$ of sgRNA were co-delivered for the silencing and reactivation experiments involving dCas9 fusions. A maximum of $1000 \mathrm{ng}$ of DNA vector was used per transfection.

Acquisition of time-lapse movies. Approximately 200,000 reporter cells stably expressing rTetR-antiGFP were seeded per well in a 24-well plate, and the next day were transfected with $1000 \mathrm{ng}$ GFP-CR plasmids using Lipofectamine LTX (Invitrogen), according to the manufacturer's instructions. We found that transfection with Lipofectamine LTX helps reduce cell death during time-lapse imaging. Six hours after transfection, cells were re-seeded at a density of $\sim 20,000$ cells in a 24-well imaging plate (Ibidi \#82406) coated with $2 \%$ Matrigel (Corning), left overnight in the incubator to adhere, and imaging was started early the next day. Imaging was done using a Leica DMi8 fluorescence microscope with Adaptive Focus Control, a $\times 20$ or $\times 40$ dry objective, and a Leica DFC 9000 GT sCMOS camera. Fluorophores were excited using a Lumencor SOLA SE II light source. Images were automatically acquired every $15 \mathrm{~min}$, using LAS X software (Leica Microsystems). Cells were grown in low-fluorescence imaging media, which consisted of FluoroBrite $^{\mathrm{TM}}$ DMEM (Gibco) supplemented with $25 \mathrm{mM}$ D-glucose (Gibco), $1 \mathrm{mM}$ sodium pyruvate (Gibco), 1× GlutaMAX ${ }^{\mathrm{TM}}$ (Gibco), 10\% Tet Approved FBS (Clontech Laboratories), and $1 \times$ Penicillin/Streptomycin (Gibco). The microscope was enclosed in an environmental control chamber (OkoLab) kept at $37^{\circ} \mathrm{C}$ and $5 \%$ $\mathrm{CO}_{2}$. Dox (Tocris) was added to the imaging media to a final concentration of $1 \mu \mathrm{g} / \mathrm{mL}$. The imaging media was changed daily for $\sim 5$ days (until the cells became too confluent to continue movies). Time-lapse movies were analyzed using ImageJ by visually tracking individual cell lineages and manually circling the area corresponding to the cell's nuclei $1 \mathrm{~h}$ after each cell division. Average fluorescence intensities of mIFP, TagRFP, and GFP within these contours of the cell nuclei were calculated and plotted based on their cell lineage using MATLAB (MathWorks).

Gene expression analysis via flow cytometry. Cells expressing stably integrated or transiently transfected rTetR-effectors were assayed by flow cytometry during and after 5 days of $1 \mu \mathrm{g} / \mathrm{mL}$ dox (Tocris) treatment. When indicated, cells were also treated with $1 \mu \mathrm{M}$ 5-Aza-2' (Sigma) or $100 \mathrm{nM}$ chaetocin (Cayman Chemical). Media containing small molecules were replaced daily. For experiments involving dCas 9 -effectors, cells were analyzed 5 days post transfection, and after being sorted for silencing (TagRFP-negative cells). On the day of flow cytometry analysis, cells were collected using $0.25 \%$ Trypsin (Life Technologies). A fraction of the cells (varying between one half to one twentieth, depending on cell density) were replated for the next time point. The remaining cells were resuspended in flow buffer ( $1 \times$ Hank's balanced salt solution (Life Technologies) and $2.5 \mathrm{mg} / \mathrm{mL}$ bovine serum albumin (BSA) (Sigma)) and filtered through a $40 \mu \mathrm{m}$ strainer (Corning) to remove cell clumps. Cellular fluorescence distributions were measured with the CytoFLEX S Flow Cytometer (Beckman Coulter) and the CytExpert Software (Beckman Coulter). The resulting data were analyzed with a custom MATLAB program called EasyFlow (https://antebilab.github.io/easyflow/). After cells were gated based on forward and side scatter, a manual gate was imposed on the TagRFP fluorescence to determine the percentage of silent cells for each sample. The gate was selected to contain $1-5 \%$ of the positive TagRFP signal in untreated cells. A total minimum of 20,000 events were recorded for each sample.

For experiments analyzing the expression of the endogenous CXCR4 gene, cells were first trypsinized and then washed with $1 \%$ BSA (Sigma) in $1 \times$ DPBS (Life Technologies). Cells were then incubated on ice for $1 \mathrm{~h}$ with monoclonal Brilliant Violet 421 (BV421)-labeled anti-CXCR4 antibody (clone 12G5 (1:20); BioLegend) BV421-labeled IgG2a (clone MOPC-173 (1:20); Biolegend) served as an isotype control. Afterwards, cells were washed three times with 1\% BSA/DPBS and then analyzed by flow cytometry for cells that were double positive for dCas 9 (mCitrine) and sgRNA (mCherry).

Targeted bisulfite sequencing. Reporter cells stably expressing rTetRantiDNMT1, rTetR-DNMT1, or rTetR-DNMT3B were treated with $1 \mu \mathrm{g} / \mathrm{mL}$ dox for 5 days. Afterwards, each treated cell line was sorted in TagRFP-negative $(+$ dox OFF) and TagRFP-positive (+dox ON) cells using a SONY SH800 Cell Sorter. Total genomic DNA was extracted from these cells with the DNeasy Blood \& Tissue Kit (Qiagen) according to the manufacturer's instructions and quantified using a NanoDrop 8000 spectrophotometer (Thermo). As a control, we also extracted DNA from cells not treated with dox (-dox) grown at the same time as the + dox cells for each cell line and sorted to include the entire population (which is $>97 \%$ ON for all cell lines, Supplementary Fig. 2b). A minimum of $2000 \mathrm{ng}$ of DNA for each sample was submitted to Zymo Research Corporation (Irvine, CA) for processing and analysis using the MethylCheck ${ }^{\mathrm{TM}}$ Service: Targeted Bisulfite Sequencing. Purified, non-methylated DNA from human HCT116 DKO cell line that contains knockouts of DNA methyltransferases, DNMT1 and DNMT3B, and in vitro-methylated DNA was obtained from Zymo Research (\#D5014).

Assays were designed targeting CpG sites in 100-300 nucleotide regions at the reporter gene and two control genes, IGF2 (positive) and ACTB (negative), using primers created with Rosefinch, Zymo Research's proprietary sodium bisulfite converted DNA-specific primer design tool. In addition, primers were designed to avoid annealing to $\mathrm{CpG}$ sites in the region of interest. In the event that $\mathrm{CpG}$ sites were absolutely necessary for target amplification, primers were synthesized with a pyrimidine ( $\mathrm{C}$ or $\mathrm{T}$ ) at the $\mathrm{CpG}$ cytosine in the forward primer, or a purine (A or $\mathrm{G}$ ) in the reverse primer to minimize amplification bias to either the methylated or unmethylated allele. A list of these primers can be found in Supplementary Table 3 Following primer validation, samples were bisulfite converted using the EZ DNA Methylation-Lightning ${ }^{\text {tw }}$ Kit (Zymo Research, \#D5030). Amplification of all samples using region-specific primer pairs was performed; the resulting amplicons were pooled and subsequently barcoded using PCR. After barcoding, samples were purified (ZR-96 DNA Clean \& Concentrator ${ }^{\mathrm{TM}}$, \#D4023) and then prepared for massively parallel sequencing using a MiSeq V2 $300 \mathrm{bp}$ Reagent Kit (Illumina) and paired-end sequencing protocol according to the manufacturer's guidelines. 
Sequence reads were identified using standard Illumina base-calling software and then analyzed using a Zymo Research proprietary analysis pipeline, which is written in Python. Low quality nucleotides and adapter sequences were trimmed off during analysis QC. Sequence reads were aligned back to the reference genome using Bismark (http://www.bioinformatics.babraham.ac.uk/projects/bismark/), an aligner optimized for bisulfite sequence data and methylation calling ${ }^{70}$. Paired-end alignment was used as default thus requiring both read 1 and read 2 be aligned within a certain distance, otherwise both read 1 and read 2 were discarded. Index files were constructed using the bismark_genome_preparation command and the entire reference genome. The --non_directional parameter was applied while running Bismark. All other parameters were set to default. Nucleotides in primers were trimmed off from amplicons during methylation calling. The methylation level of each sampled cytosine was estimated as the number of reads reporting a $\mathrm{C}$, divided by the total number of reads reporting a $\mathrm{C}$ or $\mathrm{T}$.

Statistical analysis. Data are displayed as individual points or as mean \pm SD, with sample size indicated in the figure legend. Statistical significance was evaluated using GraphPad Prism 8. A one-way analysis of variance with post hoc Tukey's honestly significant difference test or Student's two-sample unpaired $t$-test were used for statistical analysis. Statistical significance of the data is indicated as follows: ${ }^{*} p<0.05,{ }^{* *} p<0.01,{ }^{* * *} p<0.001,{ }^{* * * * *} p<0.0001 ; \mathrm{ns}=$ not significant.

Gene control modeling and data fitting. The percentage of cells in each of the 3 states in the gene control model (Fig. 4d) was derived by solving the differential equations associated with the kinetic model (Supplementary Note), and all fits were done based on these equations, as follows:

In Figs. $2 \mathrm{e}$ and $3 \mathrm{~b}$, d, and Supplementary Figs. $3 \mathrm{~b}$ and $5 \mathrm{a}$, the percentages of cells silent during the release period $\tau$ are fit to an exponential decay:

$$
S(\tau)=\left(100-I_{1}\right) e^{-k_{\mathrm{A}} \tau}+I_{1},
$$

where $I_{1}$ is the percentage of cells irreversibly silenced at the end of the recruitment period, and $k_{\mathrm{A}}$ is the rate of reactivation. A list of these rates can be found in Supplementary Table 4.

For Fig. $4 c$, we fit the fraction of cells active $(A=(1-$ fraction cells silenced $) / 100)$ after a recruitment signal ( + dox) of duration $t$ and reactivation period $(-$ dox $)$ of duration $\tau$, to the following equation (derived in Supplementary Note):

$$
A(t, \tau)=\frac{k_{\mathrm{I}}}{k_{\mathrm{I}}-k_{\mathrm{S}}} e^{-k_{\mathrm{S}}\left(t-T_{\mathrm{hg}}\right)}-\frac{k_{\mathrm{S}}}{k_{\mathrm{I}}-k_{\mathrm{S}}} e^{-k_{\mathrm{I}}\left(t-T_{\mathrm{hg}}\right)}-\frac{k_{\mathrm{S}}}{k_{\mathrm{I}}-k_{\mathrm{S}}}\left(e^{-k_{\mathrm{S}}\left(t-T_{\mathrm{lag}}\right)}-e^{-k_{\mathrm{I}}\left(t-T_{\mathrm{hg}}\right)}\right) e^{-k_{\mathrm{A}} \tau}
$$

for all recruitment times $t \geq T_{\mathrm{lag}}$, and $A(t, \tau)=1$ for $t<T_{\mathrm{lag}}$. to:

Note that for silencing time points, since $\tau=0$, the equation above simplifies

$$
A(t)=e^{-k_{\mathrm{S}}\left(t-T_{\mathrm{lag}}\right)},
$$

All experimental data points from three independent replicates across 14 days of silencing (red points in Fig. 4c, top) and 30 days of reactivation (black points in Fig. 4c, top) were recorded in MATLAB as three vectors: $A$ (percent of cells silent), $t$ (time with dox), and $\tau$ (time after dox removal). For the silencing data points $(+$ dox $) t=0,1, \ldots, 14$ and $\tau=0,0, \ldots, 0$. For reactivation data points ( - dox $)$ $t=14,14, \ldots, 14$ and $\tau=0,1,2, \ldots, 30$. These data were fit to the $A(t, \tau)$ eq. (2) above using MATLAB's fit function with the NonlinearLeastSquares method, with $k_{\mathrm{S}}, k_{\mathrm{I}}, k_{\mathrm{A}}$, and $T_{\text {lag }}$ as free parameters.

For pulsing experiments predictions in Fig. $4 \mathrm{f}$, g, the full solutions $A(t, \tau), R(t, \tau)$, and $I(t, \tau)$ (Supplementary Note) were used to calculate the fractions of cells in each state for each period. For the first + dox period, the starting conditions were $A_{0}=1, R_{0}=0, I_{0}=0$, and cells started silencing after a lag period $T_{\text {lag }}$, so we used eq. (4) in Supplementary Note. For the next periods, we applied the general eq. (3) in Supplementary Note, using as starting conditions for each period the end points from the previous one. The $T_{\text {lag }}$ period was only applied in the first dox induction period.

The linear fit in Fig. $4 \mathrm{~b}$ was performed using MATLAB's polyfit function with coefficient 1 and the goodness of fit was calculated using

$$
R^{2}=1-\frac{\left(y-y_{\text {fit }}\right)^{2}}{\operatorname{Var}(y)(N-1)},
$$

where $y$ is the mean percent cells silenced at each time point from three replicates and $N$ is the number of time points.

Reporting summary. Further information on research design is available in the Nature Research Reporting Summary linked to this article.

\section{Data availability}

All data generated during this study are included in this manuscript and available from the corresponding author upon request. Data showing protein interactions listed in Supplementary Table 1 are available from the NCBI gene database. Targeted bisulfite sequencing data are available from the NCBI SRA database (Accession number PRJNA684608). Source data are provided with this paper.

\section{Code availability}

The custom Matlab code used to plot and analyze the flow cytometry data, EasyFlow, is freely available at https://antebilab.github.io/easyflow/. The Matlab code used to fit data to the equations presented in the "Gene control modeling and data fitting" section of "Methods" is available upon request.

Received: 10 August 2020; Accepted: 15 December 2020; Published online: 22 January 2021

\section{References}

1. Gilbert, L. A. et al. CRISPR-mediated modular RNA-guided regulation of transcription in eukaryotes. Cell 154, 442-451 (2013).

2. Schultz, D. C., Friedman, J. R. \& Rauscher, F. J. 3rd Targeting histone deacetylase complexes via KRAB-zinc finger proteins: the PHD and bromodomains of KAP-1 form a cooperative unit that recruits a novel isoform of the Mi-2alpha subunit of NuRD. Genes Dev. 15, 428-443 (2001).

3. Schultz, D. C., Ayyanathan, K., Negorev, D., Maul, G. G. \& Rauscher, F. J. 3rd SETDB1: a novel KAP-1-associated histone H3, lysine 9-specific methyltransferase that contributes to HP1-mediated silencing of euchromatic genes by KRAB zinc-finger proteins. Genes Dev. 16, 919-932 (2002).

4. Yeo, N. C. et al. An enhanced CRISPR repressor for targeted mammalian gene regulation. Nat. Methods 15, 611-616 (2018).

5. Amabile, A. et al. Inheritable silencing of endogenous genes by hit-and-run targeted epigenetic editing. Cell 167, 219-232.e14 (2016).

6. O'Geen, H. et al. Ezh2-dCas9 and KRAB-dCas9 enable engineering of epigenetic memory in a context-dependent manner. Epigenetics Chromatin 12, 26 (2019).

7. Mlambo, T. et al. Designer epigenome modifiers enable robust and sustained gene silencing in clinically relevant human cells. Nucleic Acids Res. 46, 4456-4468 (2018).

8. Kwon, D. Y., Zhao, Y.-T., Lamonica, J. M. \& Zhou, Z. Locus-specific histone deacetylation using a synthetic CRISPR-Cas9-based HDAC. Nat. Commun. 8, 15315 (2017).

9. Hilton, I. B. et al. Epigenome editing by a CRISPR-Cas9-based acetyltransferase activates genes from promoters and enhancers. Nat. Biotechnol. 33, 510-517 (2015).

10. Cano-Rodriguez, D. et al. Writing of $\mathrm{H} 3 \mathrm{~K} 4 \mathrm{Me} 3$ overcomes epigenetic silencing in a sustained but context-dependent manner. Nat. Commun. 7, 12284 (2016).

11. Kearns, N. A. et al. Functional annotation of native enhancers with a Cas9histone demethylase fusion. Nat. Methods 12, 401-403 (2015).

12. Jacobs, S. A. et al. Specificity of the HP1 chromo domain for the methylated N-terminus of histone H3. EMBO J. 20, 5232-5241 (2001).

13. Platero, J. S., Hartnett, T. \& Eissenberg, J. C. Functional analysis of the chromo domain of HP1. EMBO J. 14, 3977-3986 (1995).

14. Cowieson, N. P., Partridge, J. F., Allshire, R. C. \& McLaughlin, P. J. Dimerisation of a chromo shadow domain and distinctions from the chromodomain as revealed by structural analysis. Curr. Biol. 10, 517-525 (2000).

15. Fuks, F., Hurd, P. J., Deplus, R. \& Kouzarides, T. The DNA methyltransferases associate with HP1 and the SUV39H1 histone methyltransferase. Nucleic Acids Res. 31, 2305-2312 (2003).

16. Gowher, H. \& Jeltsch, A. Molecular enzymology of the catalytic domains of the Dnmt3a and Dnmt3b DNA methyltransferases. J. Biol. Chem. 277, 20409-20414 (2002).

17. Lin, L. et al. Genome-wide determination of on-target and off-target characteristics for RNA-guided DNA methylation by dCas9 methyltransferases. Gigascience 7, 1-19 (2018).

18. Margot, J. B. et al. Structure and function of the mouse DNA methyltransferase gene: Dnmtl shows a tripartite structure. J. Mol. Biol. 297, 293-300 (2000).

19. Ma, D., Peng, S., Huang, W., Cai, Z. \& Xie, Z. Rational design of Mini-Cas9 for transcriptional activation. ACS Synth. Biol. 7, 978-985 (2018).

20. Zetsche, B., Volz, S. E. \& Zhang, F. A split-Cas9 architecture for inducible genome editing and transcription modulation. Nat. Biotechnol. 33, 139-142 (2015).

21. Truong, D.-J. J. et al. Development of an intein-mediated split-Cas9 system for gene therapy. Nucleic Acids Res. 43, 6450-6458 (2015).

22. Chew, W. L. et al. A multifunctional AAV-CRISPR-Cas 9 and its host response. Nat. Methods 13, 868-874 (2016).

23. Adli, M. The CRISPR tool kit for genome editing and beyond. Nat. Commun. 9, 1911 (2018).

24. Hassanzadeh-Ghassabeh, G., Devoogdt, N., De Pauw, P., Vincke, C. \& Muyldermans, S. Nanobodies and their potential applications. Nanomedicine 8, 1013-1026 (2013).

25. Beghein, E. \& Gettemans, J. Nanobody technology: a versatile toolkit for microscopic imaging, protein-protein interaction analysis, and protein function exploration. Front. Immunol. 8, 446 (2017).

26. Rothbauer, U. et al. Targeting and tracing antigens in live cells with fluorescent nanobodies. Nat. Methods 3, 887-889 (2006). 
27. Kirchhofer, A. et al. Modulation of protein properties in living cells using nanobodies. Nat. Struct. Mol. Biol. 17, 133-138 (2010).

28. Rothbauer, U. et al. A versatile nanotrap for biochemical and functional studies with fluorescent fusion proteins. Mol. Cell. Proteomics 7, 282-289 (2008).

29. Joshi, P. et al. The functional interactome landscape of the human histone deacetylase family. Mol. Syst. Biol. 9, 672 (2013).

30. Hathaway, N. A. et al. Dynamics and memory of heterochromatin in living cells. Cell 149, 1447-1460 (2012).

31. Zhang, C. L., McKinsey, T. A. \& Olson, E. N. Association of class II histone deacetylases with heterochromatin protein 1: potential role for histone methylation in control of muscle differentiation. Mol. Cell. Biol. 22, 7302-7312 (2002).

32. Minc, E., Courvalin, J.-C. \& Buendia, B. HP1 $\gamma$ associates with euchromatin and heterochromatin in mammalian nuclei and chromosomes. Cytogenet. Genome Res. 90, 279-284 (2000).

33. Vakoc, C. R., Mandat, S. A., Olenchock, B. A. \& Blobel, G. A. Histone H3 lysine 9 methylation and HP1gamma are associated with transcription elongation through mammalian chromatin. Mol. Cell 19, 381-391 (2005).

34. Lomberk, G., Bensi, D., Fernandez-Zapico, M. E. \& Urrutia, R. Evidence for the existence of an HP1-mediated subcode within the histone code. Nat. Cell Biol. 8, 407-415 (2006).

35. Moutel, S. et al. NaLi-H1: a universal synthetic library of humanized nanobodies providing highly functional antibodies and intrabodies. Elife 5, e16228 (2016).

36. Nishiyama, A. et al. Two distinct modes of DNMT1 recruitment ensure stable maintenance DNA methylation. Nat. Commun. 11, 1222 (2020).

37. O'Leary, V. B. et al. Long non-coding RNA PARTICLE bridges histone and DNA methylation. Sci. Rep. 7, 1790 (2017).

38. Margolin, J. F. et al. Kruppel-associated boxes are potent transcriptional repression domains. Proc. Natl Acad. Sci. USA 91, 4509-4513 (1994).

39. Braun, S. M. G. et al. Rapid and reversible epigenome editing by endogenous chromatin regulators. Nat. Commun. 8, 560 (2017).

40. Bintu, L. et al. Dynamics of epigenetic regulation at the single-cell level. Science 351, 720-724 (2016).

41. O'Geen, H. et al. dCas9-based epigenome editing suggests acquisition of histone methylation is not sufficient for target gene repression. Nucleic Acids Res. 45, 9901-9916 (2017).

42. Liu, X. S. et al. Editing DNA methylation in the mammalian genome. Cell 167, 233-247.e17 (2016).

43. Vojta, A. et al. Repurposing the CRISPR-Cas9 system for targeted DNA methylation. Nucleic Acids Res. 44, 5615-5628 (2016).

44. Chedin, F., Lieber, M. R. \& Hsieh, C.-L. The DNA methyltransferase-like protein DNMT3L stimulates de novo methylation by Dnmt3a. Proc. Natl Acad. Sci. USA 99, 16916-16921 (2002).

45. Siddique, A. N. et al. Targeted methylation and gene silencing of VEGF-A in human cells by using a designed Dnmt3a-Dnmt3L single-chain fusion protein with increased DNA methylation activity. J. Mol. Biol. 425, 479-491 (2013).

46. Stepper, P. et al. Efficient targeted DNA methylation with chimeric dCas9Dnmt3a-Dnmt3L methyltransferase. Nucleic Acids Res. 45, 1703-1713 (2017).

47. Dalchau, N. et al. Computing with biological switches and clocks. Nat. Comput. 17, 761-779 (2018).

48. Larson, A. G. et al. Liquid droplet formation by HPla suggests a role for phase separation in heterochromatin. Nature https://doi.org/10.1038/nature22822 (2017).

49. Strom, A. R. et al. Phase separation drives heterochromatin domain formation. Nature 547, 241-245 (2017).

50. Grozinger, C. M. \& Schreiber, S. L. Regulation of histone deacetylase 4 and 5 and transcriptional activity by 14-3-3-dependent cellular localization. Proc. Natl Acad. Sci. USA 97, 7835-7840 (2000).

51. Wang, A. H. et al. Regulation of histone deacetylase 4 by binding of 14-3-3 proteins. Mol. Cell. Biol. 20, 6904-6912 (2000).

52. Kao, H. Y. et al. Mechanism for nucleocytoplasmic shuttling of histone deacetylase 7. J. Biol. Chem. 276, 47496-47507 (2001).

53. Roberts, B. et al. Systematic gene tagging using CRISPR/Cas9 in human stem cells to illuminate cell organization. Mol. Biol. Cell 28, 2854-2874 (2017).

54. Harikumar, A. et al. An endogenously tagged fluorescent fusion protein library in mouse embryonic stem cells. Stem Cell Rep. 9, 1304-1314 (2017)

55. Leonetti, M. D., Sekine, S., Kamiyama, D., Weissman, J. S. \& Huang, B. A scalable strategy for high-throughput GFP tagging of endogenous human proteins. Proc. Natl Acad. Sci. USA 113, E3501-E3508 (2016).

56. Götzke, H. et al. The ALFA-tag is a highly versatile tool for nanobody-based bioscience applications. Nat. Commun. 10, 4403 (2019).

57. Braun, M. B. et al. Peptides in headlock - a novel high-affinity and versatile peptide-binding nanobody for proteomics and microscopy. Sci. Rep. 6, 19211 (2016).

58. Virant, D. et al. A peptide tag-specific nanobody enables high-quality labeling for dSTORM imaging. Nat. Commun. 9, 930 (2018).

59. De Genst, E. J. et al. Structure and properties of a complex of $\alpha$-synuclein and a single-domain camelid antibody. J. Mol. Biol. 402, 326-343 (2010).
60. Ling, J. et al. A nanobody that recognizes a 14-residue peptide epitope in the E2 ubiquitin-conjugating enzyme UBC6e modulates its activity. Mol. Immunol. 114, 513-523 (2019).

61. McDonald, J. I. et al. Reprogrammable CRISPR/Cas9-based system for inducing site-specific DNA methylation. Biol. Open 5, 866-874 (2016).

62. Galonska, C. et al. Genome-wide tracking of dCas9-methyltransferase footprints. Nat. Commun. 9, 597 (2018)

63. $\mathrm{Xu}, \mathrm{X}$. et al. A CRISPR-based approach for targeted DNA demethylation. Cell Discov. 2, 16009 (2016).

64. Choudhury, S. R., Cui, Y., Lubecka, K., Stefanska, B. \& Irudayaraj, J. CRISPRdCas9 mediated TET1 targeting for selective DNA demethylation at BRCA1 promoter. Oncotarget 7, 46545-46556 (2016).

65. Bekesi, A. et al. in Methods in Enzymology (ed. Rhoades, E.) vol. 611, 607-675 (Academic Press, 2018).

66. Tycko, J. et al. Mitigation of off-target toxicity in CRISPR-Cas9 screens for essential non-coding elements. Nat. Commun. 10, 4063 (2019).

67. Gao, Y. et al. Complex transcriptional modulation with orthogonal and inducible dCas9 regulators. Nat. Methods 13, 1043-1049 (2016).

68. Ghosh, R. P. et al. A fluorogenic array for temporally unlimited singlemolecule tracking. Nat. Chem. Biol. 15, 401-409 (2019).

69. Gilbert, L. A. et al. Genome-scale CRISPR-mediated control of gene repression and activation. Cell 159, 647-661 (2014).

70. Krueger, F. \& Andrews, S. R. Bismark: a flexible aligner and methylation caller for Bisulfite-Seq applications. Bioinformatics 27, 1571-1572 (2011).

\section{Acknowledgements}

This work was supported by NIH MIRA R35GM128947-01 (L.B.), NIH T32 Training Grant T32GM007276 (M.V.V.), a Burroughs Wellcome Fund Career Award at the Scientific Interface (L.B.), and a Japan Society for the Promotion of Science Overseas Research Fellowship (T.F.). We thank Frank Perez and Sandrine Moutel (Institut Curie) for the antiHP1 nanobody plasmid, Rajarshi Ghosh and Jan Liphardt (Stanford University) for the antiGFP nanobody plasmid, Stanley Qi (Stanford University) and members of his lab for allowing us to use their flow cytometer, Josh Tycko and Joydeb Sinha (Stanford University) for providing us with plasmids, and members of the Bintu Lab for useful feedback on the manuscript.

\section{Author contributions}

M.V.V. and L.B. designed the study. M.V.V. generated the DNA constructs and cell lines, and performed the flow cytometry experiments and associated data analysis. T.F. performed the experiments and data analysis for the time-lapse movies. L.B. derived and fit the threestate gene control model. M.V.V. and L.B. wrote the manuscript with inputs from T.F.

\section{Competing interests}

The authors declare no competing interests.

\section{Additional information}

Supplementary information is available for this paper at https://doi.org/10.1038/s41467020-20757-1.

Correspondence and requests for materials should be addressed to L.B.

Peer review information Nature Communications thanks Ross Cheloha and the other, anonymous, reviewer for their contribution to the peer review of this work. Peer reviewer reports are available.

Reprints and permission information is available at http://www.nature.com/reprints

Publisher's note Springer Nature remains neutral with regard to jurisdictional claims in published maps and institutional affiliations.

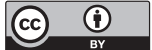

Open Access This article is licensed under a Creative Commons Attribution 4.0 International License, which permits use, sharing, adaptation, distribution and reproduction in any medium or format, as long as you give appropriate credit to the original author(s) and the source, provide a link to the Creative Commons license, and indicate if changes were made. The images or other third party material in this article are included in the article's Creative Commons license, unless indicated otherwise in a credit line to the material. If material is not included in the article's Creative Commons license and your intended use is not permitted by statutory regulation or exceeds the permitted use, you will need to obtain permission directly from the copyright holder. To view a copy of this license, visit http://creativecommons.org/ licenses/by/4.0/.

(C) The Author(s) 2021 